\title{
Laboreal
}

Volume $14 \mathrm{~N}^{\circ} 1$ | 2018

O regresso ao emprego após um acidente de trabalho

\section{O espaço experiencial da dor: sofrimento, corpo e inscrição social depois do acidente de trabalho entre trabalhadores industriais portugueses}

El espacio experiencial del dolor : sufrimiento, cuerpo e inscripción social tras un accidente de trabajo entre trabajadores industriales portugueses

L'espace expérientiel de la douleur: souffrance, corps et inscription sociale après l'accident de travail parmi les travailleurs industriels portugais

The experiential spectrum of pain: suffering, body and social inscription after the work accident among Portuguese industrial workers

\section{Bruno Monteiro e Virgílio Borges Pereira}

\section{OpenEdition}

\section{Journals}

Edição electrónica

URL: http://journals.openedition.org/laboreal/519

DOI: $10.4000 /$ laboreal.519

ISSN: 1646-5237

Editora

Universidade do Porto

Refêrencia eletrónica

Bruno Monteiro e Virgílio Borges Pereira, « O espaço experiencial da dor: sofrimento, corpo e inscrição social depois do acidente de trabalho entre trabalhadores industriais portugueses », Laboreal [Online] Volume $14 \mathrm{~N}^{0} 1$ | 2018, posto online no dia 01 julho 2018, consultado o 07 outubro 2019. URL : http:// journals.openedition.org/laboreal/519; DOI : 10.4000/laboreal.519

Este documento foi criado de forma automática no dia 7 outubro 2019

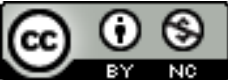

Laboreal está licenciado com uma Licença Creative Commons - Atribuição-NãoComercial 4.0 Internacional. 


\section{O espaço experiencial da dor: sofrimento, corpo e inscrição social depois do acidente de trabalho entre trabalhadores industriais portugueses}

El espacio experiencial del dolor : sufrimiento, cuerpo e inscripción social tras un accidente de trabajo entre trabajadores industriales portugueses

L'espace expérientiel de la douleur: souffrance, corps et inscription sociale après l'accident de travail parmi les travailleurs industriels portugais

The experiential spectrum of pain: suffering, body and social inscription after the work accident among Portuguese industrial workers

Bruno Monteiro e Virgílio Borges Pereira

\section{NOTA DO EDITOR}

http://dx.doi.org/10.15667/laborealxiv0118bm

Manuscrito recebido em : janeiro/2018

Aceite após peritagem : junho/2018

\section{Introdução : uma «guerra civil» invisível em Portugal}

Ao longo dos treze anos compreendidos entre 2000 e 2021, teve lugar uma «guerra civil» em Portugal, somando 3196 vítimas mortais. Dando uma tangibilidade inesperada à expressão utilizada por Karl Marx, este número é apenas a expressão mais dramática dos acidentes de trabalho ocorridos durante aquele período de tempo, compreendendo 
de 215632 a 248097 acidentes todos os anos e implicando uma perda anual entre 6088 165 e 7624893 dias de trabalho. O impacto do acidente sobre as dimensões pessoais, as práticas quotidianas e o apoio institucional vê-se, por vezes, estudado em separado, isolando cada uma dessas vertentes em pesquisas parciais e parcelares. A investigação integrada sobre a sua imbricação comum numa mesma trajetória biográfica - por outras palavras, num mesmo corpo socializado (Wacquant, 1998) - apela para uma " sociologia de indivíduos » mais do que para uma «sociologia de variáveis». Usando os materiais empíricos reunidos num recente projeto de investigação, este artigo procura apresentar o espaço experiencial da dor que se constitui para distintos grupos de trabalhadores industriais que sofreram acidentes de trabalho em Portugal. A partir deste ponto de vista analítico, podemos visualizar as associações e contrastes estabelecidos entre as dimensões da dor aparentemente mais «pessoais», os dispositivos institucionais usados para o tratamento e a reabilitação, e as redes e práticas que sustentam a vida prosaica destes trabalhadores depois da ocorrência de um acidente laboral. Neste artigo, tentou-se suplementar a leitura relacional dos dados estatísticos que informa esta abordagem sociológica com os contributos trazidos pelas observações etnográficas e epidemiológicas que interrogam as vertentes sociais e culturais da incorporação, até nos seus aspetos mais imediatamente tidos por «biológicos» (Lock and Kaufert, 2001 ; Scheper-Hughes and Lock, 1987; Holmes, 2013). Deste modo, procurou-se renovar a rearticulação entre as dimensões «objetivas» e «subjectivas» das consequências biográficas pós-sinistro, que permanecem incomunicáveis entre elas quando são analiticamente distribuídas por distintas especializações académicas e científicas.[1]

\section{Acidentes de trabalho, experiências de dor e situação socioinstitucional}

2 Lidar com uma noção carregada de preconceitos de senso comum, como sucede com «acidente de trabalho», implica a concretização de ruturas epistemológicas abruptas ou, vendo-as de outro modo, aproveitar os próprios obstáculos que se colocam durante a pesquisa como oportunidades para desafiar os limites assumidos, ainda que inconscientemente, a respeito do desenvolvimento de uma sociologia da incorporação dos acidentes de trabalho alinhada com os incentivos trazidos por uma epidemiologia social (vd. Krieger, 2005). Por razões de concisão, vamos selecionar um grupo de quarto princípios de interpretação sobre acidentes que trabalho que, especialmente resilientes entre as conceções populares e eruditas a seu respeito, mereceram ser desafiadas desde o início da nossa pesquisa. Primeiro, o acidente não é o fim da história. No entanto, quando comparado com a pletora de estudos sobre as determinantes sociais e ecológicas dos acidentes de trabalho, o número de pesquisas sobre o pós-sinistro é mais escasso (Ghisi et al, 2012). Tem grande relevância o estudo das consequências sociais, institucionais e pessoais que derivam da ocorrência de um acidente de trabalho, que tende a deslizar para a «invisibilidade» ainda que seja literalmente incorporado pelo trabalhador sinistrado (Daubas-Letourneux, 2012). Há «mais do que o olho avista» para além do horizonte do acidente, incluindo implicações que são físicas e mentais, tal como são sociais e emocionais (Lax and Klein, 2008).

3 Segundo, a própria trajetória pós-acidente está estruturada por vetores sociológicos que explicam variações na magnitude da dor, no enquadramento institucional da 
reabilitação ou reinserção, ou nas redes de sociação - em suma, as consequências do acidente estão longe de ser eles próprias acidentais em termos sociais (Desmarez, Godin e Renneson, 2007). Em oposição a uma «des-socialização» da própria noção de acidente de trabalho (Renoir, 1980), é importante sublinhar a necessidade de suplementar as interpretações ergonómicas, tecnológicas ou psicológicas por meio da ênfase sobre o encastramento sociológico dos trabalhadores sinistrados.

Terceiro, as reverberações biográficas do acidente de trabalho não significam tomar individualmente os trabalhadores como recetores passivos de um «destino». São desenvolvidas estratégias para lidar com o acidente e as suas implicações, ainda que de maneira sensível aos atributos sociais, culturais e económicos que caracterizam os trabalhadores em questão - uma circunstância que pode dobrar o viés inicial criado pela estruturação social sobre a incidência assimétrica de acidentes de trabalho entre as distintas ocupações e setores. Aliás, a disponibilidade e as utilizações das «tecnologias de cuidado» são elas próprias condicionadas por tais mediações (Lovelock, 2012).

Por último, existe uma limitação especificamente analítica, nascida de tradições disciplinares que tendem a excluir entre si o social e o biológico, o económico e o interpessoal, o psicológico e o institucional como componentes independentes dentro do universo das pesquisas sobre o pós-sinistro (Dembe, 2001). Daqui surge a necessidade de desenvolver uma abordagem teórico-metodológica que sobreponha as lentes física, psicológica e sociológica no estudo das trajetórias biográficas subsequentes ao acidente de trabalho, incluindo os processos de regresso ao trabalho (Franche e Krause, 2002). A presente pesquisa foi uma tentativa para mostrar, pela mera composição da equipa de investigação, que é possível superar na prática os cânones mais consagrados que separam sociologia e psicologia - e, deste modo, resgatar o projeto maussiano de uma sua imbricação (Mauss, 1969). Tendo este propósito mais abrangente, este artigo procura precisamente construir e explorar o espaço experiencial da dor que nasce de um acidente de trabalho.

\section{Método}

O projeto de investigação 'Regresso ao trabalho depois do acidente : superar obstáculos' foi promovido pela Associação Nacional dos Deficientes Sinistrados no Trabalho (ANDST), com o apoio do Instituto Nacional para a Reabilitação, entre Março e Novembro de 2016. A equipa de investigação era constituída por sociólogos e psicólogos do trabalho, visando estabelecer uma plataforma comum que fosse capaz de exibir, discutir e, desejavelmente, corrigir os enviesamentos, limitações ou desconhecimentos mútuos decorrentes de culturas de conhecimento específicas e das próprias divisões do trabalho científico ('social' vs. 'individual', 'objetivo' vs. 'subjetivo', ...), enquanto beneficiava de uma combinação de heranças paradigmáticas e das respetivas técnicas de inquirição. $O$ projeto teve como peça central a aplicação de um inquérito por questionário a sinistrados do trabalho. A construção deste beneficiou da realização prévia de 12 entrevistas em profundidade, que permitiram a reconstrução das trajetórias biográficas e institucionais de indivíduos sinistrados em acidentes de trabalho. Juntamente com a revisão de investigações prévias sobre o tema, estas entrevistas foram usadas para selecionar os elementos sociais e profissionais críticos para a comparação das situações dos entrevistados antes e depois do acidente, para 
mapear os enquadramentos institucionais durante os processos de reabilitação e de reparação e para identificar os aspetos pessoais e coletivos da fase pós-acidente contemplados no desenho do inquérito.

O inquérito continha 53 questões, divididas por três núcleos principais de pesquisa : caracterização sociográfica; circunstâncias e consequências do acidente; e a vida quotidiana depois do acidente. Baseado num diálogo com investigação sociológica anterior sobre a estruturação do território português (Ferrão, 2017) e sobre o significado das divisões sociais e da sua relevância nos acidentes de trabalho (Pinto e Queiroz, 1990 ; Pinto, 2000), o inquérito deu prioridade à recolha de informação sociográfica sobre o sexo, a idade, o estatuto marital, a composição familiar, a escolarização, o local de nascimento e de residência, a situação laboral, o desemprego, assim como sobre a profissão atual dos respondentes. Privilegiando o estudo enraizado das circunstâncias e consequências do acidente (Burdof et al., 1997; Dembe, 2001, Keller, 2001), o inquérito permitiu a reunião de informação significativa sobre os acidentes dos inquiridos, incluindo medidas extensivas sobre os impactos físicos e psicológicos nos indivíduos, como o Nottingham Health Profile [2], e o apoio institucional que receberam. Para além da descrição do acidente, o inquérito reteve elementos adicionais sobre a situação social dos inquiridos antes e depois do acidente - tipo de incapacidade, fonte de rendimento, profissão e calendário de trabalho diário, entre outras informações, foram especificamente documentadas no inquérito. $O$ estudo da (re)constituição da vida quotidiana dos inquiridos depois do acidente, inspirado por resultados anteriores de pesquisas sobre o quotidiano de trabalhadores portugueses (Monteiro, 2014 ; Pereira, 2011), envolveu o inventário de informação sobre a dinâmica familiar, o tempo de lazer, as práticas de sociabilidade e as perspetivas sobre o futuro pessoal.

8 Foi, assim, construída uma amostra nacional representativa de população sinistrada no trabalho. Esta reteve o género e a região como critérios de representatividade, de acordo com a distribuição do número de acidentes de trabalho registada nas últimas estatísticas nacionais disponíveis à data sobre acidentes de trabalho (2010). A seleção dos casos individuais foi realizada, de modo aleatório, a partir da base de dados da própria ANDST, com a exceção de casos de associados com mais de dez anos de inscrição, uma vez que tal circunstância poderia impedir um relato detalhado do acidente. Através do estabelecimento de limiares para as dimensões de género e regionais consideradas, os potenciais enviesamentos contidos na base de dados revista (4097 indivíduos) foram controlados, pelo menos, nestas dimensões. O género e a região foram retidos como variáveis de seleção para a estruturação da amostra por serem as informações mais exaustivas disponíveis na base de dados da Associação. Não obstante limitações intrínsecas, esta opção assegurou a robustez e a confiança necessária à utilização da amostra, para além de permitir um uso adequado dos recursos limitados disponíveis para o desenvolvimento deste projeto de investigação. Os detalhes sobre a gravidade do acidente eram de difícil recolha, ou encontravam-se incompletos, na base de dados da Associação, razões que estiveram subjacentes à respetiva não inclusão na construção da amostra. $O$ mesmo se aplica às diferentes ocupações profissionais. As definições das profissões dos membros no momento do acidente estavam registadas de modo impreciso ou equívoco na base de dados, tornando, por isso, arriscada qualquer tentativa de as agregar em categorias mais vastas - na verdade, foi apenas o inquérito que permitiu determinar com maior precisão as profissões dos inquiridos. 
O inquérito permitiu a reunião de informação relativa a um total de 366 indivíduos, de acordo com um procedimento que fora desenhado para ultrapassar quer as limitações do projeto (urgência temporal, equipa de pesquisa com apenas dois investigadores no terreno juniores a tempo parcial, restrições financeiras) quer as limitações decorrentes de uma população a inquirir geograficamente dispersa e fisicamente fragilizada (e, frequentemente, constrangida economicamente, dados os perfis sociais detetados durante os contatos). Depois da seleção de casos elegíveis para o inquérito, um contato telefónico com os potenciais inquiridos era estabelecido com o objetivo de explicar as ambições do inquérito e o pedido de colaboração efetuado ; quando a colaboração era objeto de aceitação, um exemplar do inquérito era enviado pelo correio com instruções e um envelope para a respetiva devolução. Uma segunda chamada telefónica era efetuada para confirmar a receção do inquérito e para dar instruções adicionais ou resolver questões decorrentes do contato com o inquérito. Beneficiando das visitas ocasionais à Associação, ou de iniciativas desta, um número mais pequeno de membros da Associação $(\mathrm{N}=40)$ foi inquirido pessoalmente sempre que preenchiam as exigências metodológicas. Nestes casos, era solicitado a colaboradores permanentes da Associação para aplicarem eles próprios o inquérito aos membros conhecidos naquelas visitas. Dos 760 inquéritos distribuídos destes dois modos, recolheram-se 366, perfazendo uma taxa de devolução de 48,2 \%. Durante a aplicação do inquérito, os colaboradores cumpriram as instruções metodológicas mais rigorosas, o que foi confirmado pela presença daqueles dois membros juniores da equipa de pesquisa de terreno nas instalações da Associação, circunstância que lhes permitiu também verificar pessoalmente todos os inquéritos recebidos pelo correio.

10 A amostra constituída pelos inquéritos válidos era fundamentalmente constituída por homens (77\%), $40 \%$ dos respondentes tinham 55 ou mais anos. Para os objetivos da pesquisa aqui apresentada, a informação reunida nos inquéritos foi usada para construir uma base de dados, submetida posteriormente a um tratamento e a uma interpretação necessários à sua leitura relacional, que apresentaremos na secção seguinte deste texto. De modo a maximizar o potencial da análise, a nossa leitura relacional focar-se-á nos inquiridos com uma ocupação profissional específica no período do acidente, retendo, em concreto, os trabalhadores que exerciam profissões enquanto operários industriais, o que corresponde a 186 inquiridos. $93 \%$ destes trabalhadores são homens. 39 \% tinham 55 ou mais anos. Dada esta concentração, optou-se por não fazer incidir a análise sobre o significado das divisões de género.

\section{As modalidades da experiência posterior ao acidente de trabalho dos operários industriais : uma leitura relacional}

11 Tomando por referência a informação apresentada na Tabela 1, desenvolvemos um procedimento iterativo que permitiu a produção de uma leitura sociológica relacional (Bourdieu, 1979) do impacto do acidente de trabalho na vida quotidiana dos operários industriais inquiridos no âmbito da pesquisa. Esta leitura relacional, informada pelos princípios da Análise Geométrica de Dados e realizada com base numa Análise de Correspondências Múltiplas [ $\left.{ }^{3}\right]$ (ACM) 'específica' [4] (Le Roux \& Rouanet, 2010), retém 26 questões, 62 modalidades, organizadas de acordo com três temas: sociabilidade quotidiana e atividades culturais depois do acidente (nove variáveis, 23 modalidades) ; a 
relação dos inquiridos com diferentes instituições com um papel na definição dos respetivos acidentes (nove variáveis ; 18 modalidades); e a leitura dos inquiridos sobre a natureza e o impacto do acidente de trabalho que sofreram (oito variáveis; 21 modalidades). A Tabela 2 apresenta os eixos, valores próprios, taxas de variância e taxas de variância modificadas da ACM 'específica', que desenvolvemos com recurso ao programa de análise e tratamento de dados SPAD versão 7. 4. A análise retém os resultados dos dois primeiros eixos, que têm uma taxa modificada de $78,36 \%$ e, como veremos, são suscetíveis de uma leitura relacional significativa tanto em termos estatísticos como sociológicos.

Tabela 1 : variáveis usadas na leitura relacional da experiência do pós-acidente de trabalho

\begin{tabular}{|c|c|c|}
\hline $\begin{array}{l}\text { Representações do impacto do } \\
\text { acidente (R) }\end{array}$ & $\begin{array}{l}\text { Práticas quotidianas após } \\
\text { acidente de trabalho } \\
\text { (sociabilidade e cultura) (P) }\end{array}$ & Apoio Institucional (I) \\
\hline $\begin{array}{l}\text { Perfil legal da incapacidade (6 } \\
\text { modalidades) }\end{array}$ & $\begin{array}{l}\text { Praticar desporto ( } 3 \text { modalidades; } \\
1 \text { passiva) }\end{array}$ & $\begin{array}{l}\text { Empresa } \\
\text { modalidades })\end{array}$ \\
\hline $\begin{array}{l}\text { Ter dor depois do acidente }(2 \\
\text { modalidades })\end{array}$ & $\begin{array}{l}\text { Jardinagem (3 modalidades ; } 1 \\
\text { passiva) }\end{array}$ & $\begin{array}{l}\text { Tribunal } \\
\text { modalidades })\end{array}$ \\
\hline $\begin{array}{l}\text { Ter medo depois do acidente ( } 2 \\
\text { modalidades) }\end{array}$ & $\begin{array}{l}\text { Tarefas domésticas } \\
\text { modalidades) }\end{array}$ & $\begin{array}{l}\text { Companhia de seguros } \\
\text { ( } 2 \text { modalidades }\end{array}$ \\
\hline $\begin{array}{l}\text { Ter vergonha depois do acidente ( } 2 \\
\text { modalidades) }\end{array}$ & $\begin{array}{l}\text { Atividades } \quad \text { sociais } \\
\text { modalidades } ; 1 \text { passiva) }\end{array}$ & $\begin{array}{l}\text { Hospital } \\
\text { modalidades) }\end{array}$ \\
\hline $\begin{array}{l}\text { Ter um mau futuro por causa do } \\
\text { acidente ( } 2 \text { modalidades) }\end{array}$ & Leitura (3 modalidades) & $\begin{array}{l}\text { Sindicato } \\
\text { modalidades })\end{array}$ \\
\hline $\begin{array}{l}\text { Sentir-se desconfortável com o } \\
\text { próprio corpo por causa do acidente } \\
\text { ( } 2 \text { modalidades) }\end{array}$ & Ir ao café ( 3 modalidades) & $\begin{array}{l}\text { Família e amigos }(2 \\
\text { modalidades })\end{array}$ \\
\hline $\begin{array}{l}\text { Reabilitação física depois do acidente } \\
\text { ( } 3 \text { modalidades ; } 1 \text { passiva) }\end{array}$ & Sair com amigos (3 modalidades) & ACT (2 modalidades) \\
\hline $\begin{array}{l}\text { Reabilitação psicológica depois do } \\
\text { acidente ( } 3 \text { modalidades) }\end{array}$ & Andar a pé ( 3 modalidades) & $\begin{array}{lr}\text { Instituto Nacional } & \text { de } \\
\text { Reabilitação } & (2 \\
\text { modalidades }) & \end{array}$ \\
\hline & Ira o cinema ( 3 modalidades) & $\begin{array}{l}\text { Segurança Social } \quad(2 \\
\text { modalidades })\end{array}$ \\
\hline Oito variáveis ; 21 modalidades & Nove variáveis ; 23 modalidades & $\begin{array}{l}\text { Nove variáveis; } 18 \\
\text { modalidades }\end{array}$ \\
\hline
\end{tabular}


Tabela 2 : Análise de Correspondências Múltiplas ‘Específica' - Eixos, Valores Próprios, Taxas de Variância e Taxas de Variância Modificada - Total dos valores próprios : 1,3564

\begin{tabular}{|l|l|l|l|}
\hline Eixos & Valores próprios & Taxas de Variância ( \%) & Taxas de Variância Modificada ( \%) \\
\hline 1 & 0,1646 & 12,13 & 50,01 \\
\hline 2 & 0,1309 & 9,65 & 28,35 \\
\hline 3 & 0,0789 & 5,09 & 6,9 \\
\hline 4 & 0,0690 & 4,71 & 4,47 \\
\hline
\end{tabular}

12 A Figura 1 apresenta a nuvem dos indivíduos dos 186 trabalhadores industriais que foram submetidos ao procedimento de análise estatística enunciado.

Figura 1 : Nuvem de indivíduos - 186 operários industriais

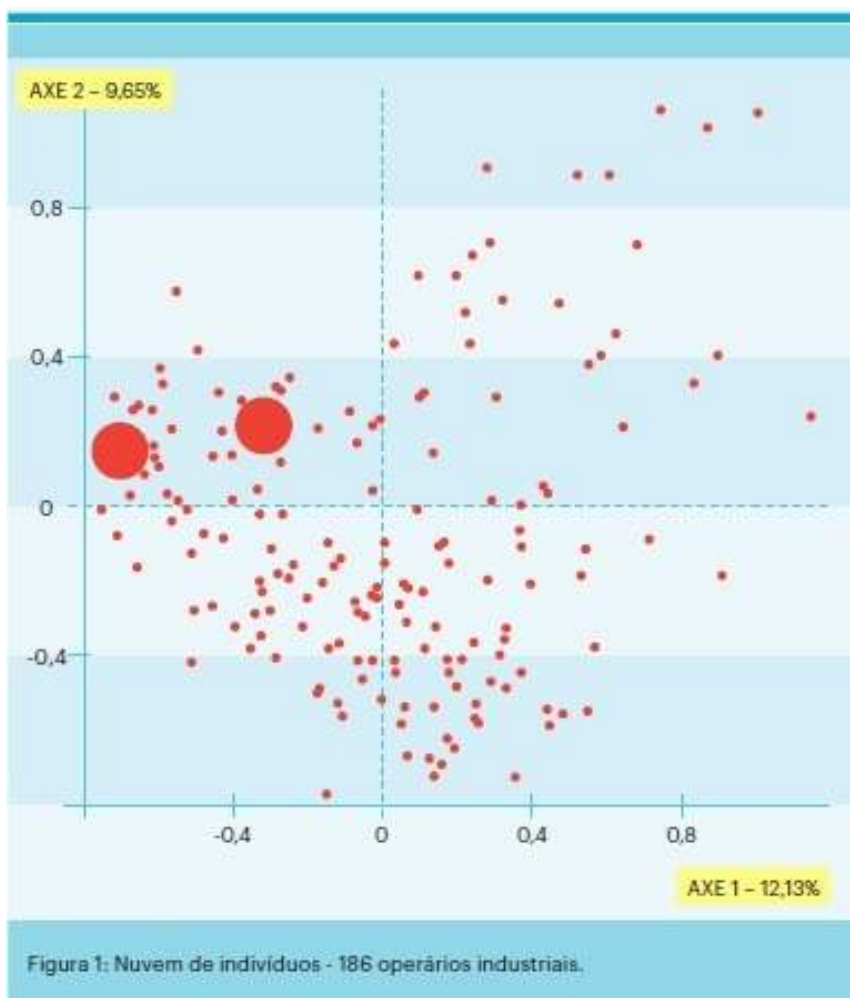

13 A Tabela 3 e a figura 1 apresentam o primeiro eixo da ACM 'específica', que tem uma taxa modificada de 50,01\%, com as suas 25 categorias mais significativas. As modalidades aqui representadas contribuem com 78,2 \% da variância do eixo. Em suma, no lado direito podemos encontrar as modalidades que dizem respeito à prática pelos operários industriais inquiridos de uma sociabilidade moderada e ao usufruto de um apoio institucional relevante (com mobilizações de diferentes instituições, i.e. Segurança Social ou Sindicatos) associadas a sentimentos acrescidos de dor (com uma contribuição global de 51,0 \% para a variância do eixo). No lado esquerdo da figura, associam-se as experiências definidas pela ausência de práticas de sociabilidade e de apoio institucional (com uma contribuição de 27,2 \% para a variância do eixo). 
Tabela 3: 25 modalidades significativas representadas na Figura 2 e respetivas contribuições para o eixo 1

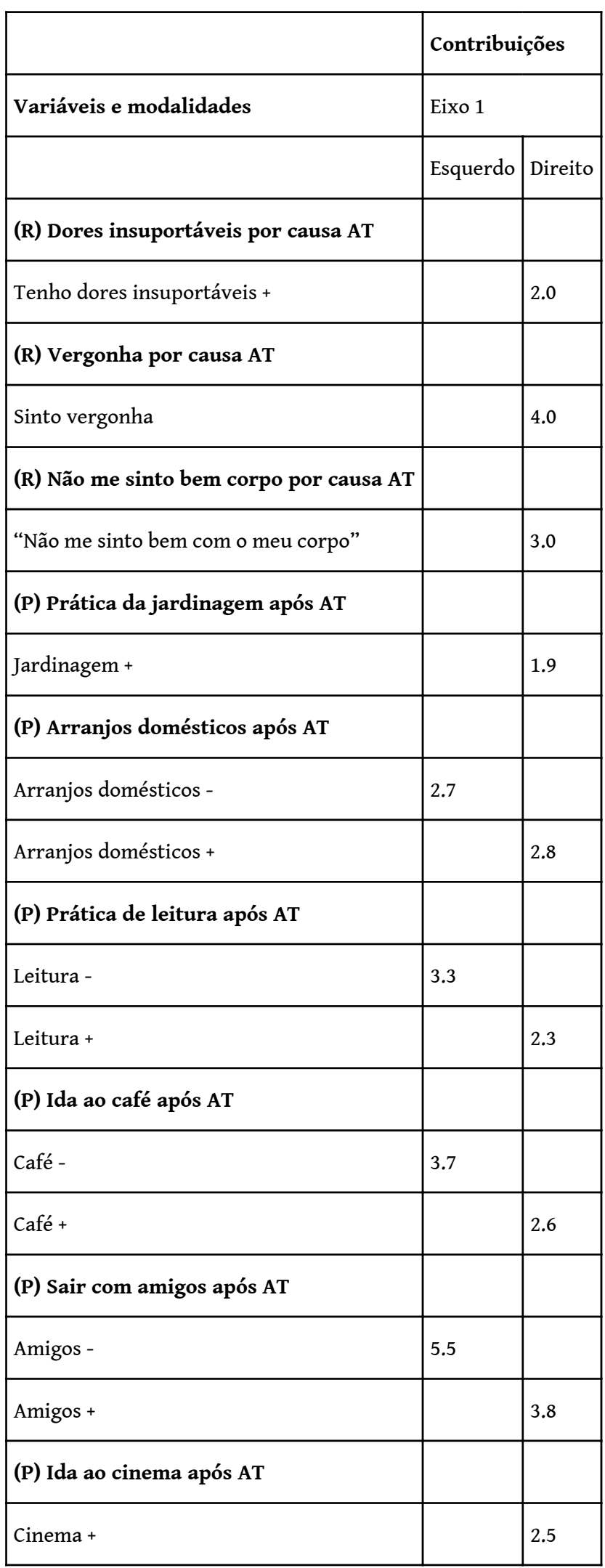




\begin{tabular}{|c|c|c|}
\hline Apoio do tribunal após & & \\
\hline Tribunal + & & 2.6 \\
\hline Tribunal - & 2.5 & \\
\hline \multicolumn{3}{|c|}{ Apoio da seguradora após AT } \\
\hline Seguradora + & & 1.8 \\
\hline Seguradora - & 2.4 & \\
\hline \multicolumn{3}{|c|}{ Apoio do hospital após AT } \\
\hline Hospital: + & & 3.8 \\
\hline Hospital - & 2.3 & \\
\hline \multicolumn{3}{|c|}{ Apoio do sindicato após AT } \\
\hline Sindicato + & & 2.5 \\
\hline \multicolumn{3}{|l|}{ Apoio familiar após AT } \\
\hline Família + & & 3.1 \\
\hline Família - & 4.8 & \\
\hline \multicolumn{3}{|l|}{ Apoio ACT após AT } \\
\hline $\mathrm{ACT}+$ & & 4.8 \\
\hline \multicolumn{3}{|l|}{ Apoio INR após AT } \\
\hline INR + & & 4.6 \\
\hline \multicolumn{3}{|c|}{ Apoio Segurança Social após AT } \\
\hline Seg. social + & & 2.9 \\
\hline Total das contribuições & 27.2 & 51.0 \\
\hline
\end{tabular}


Figura 2 : ACM 'específica. Nuvem das categorias - 25 modalidades com maiores contribuições para o eixo 1

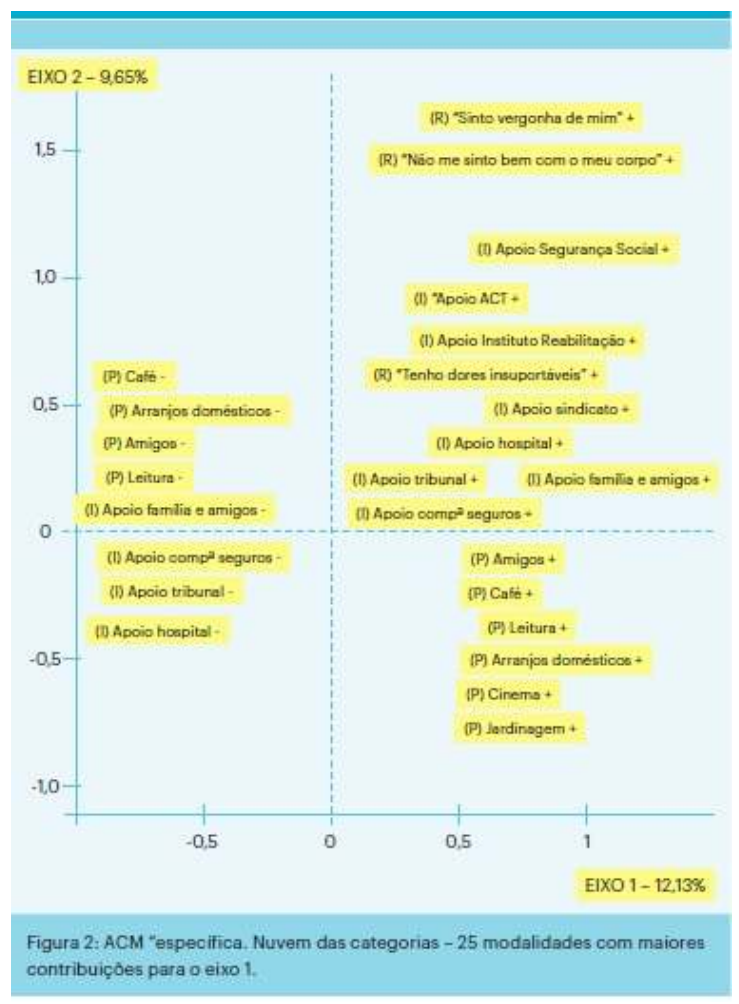

14 A Tabela 4 e a Figura 3 representam os resultados do segundo eixo 'específico', que tem uma taxa modificada de $28,35 \%$, com as suas 24 categorias mais significativas. As modalidades aqui em questão contribuem com 85,2 \% da variância do eixo. No lado superior do eixo, para além da presença de sentimentos de medo, de vergonha e de dor, que se acompanharam de um seguimento psicológico, podemos identificar a associação entre estas leituras e o reconhecimento da importância do apoio institucional obtido no Sindicato ou na Segurança Social, assim como o significado da ausência de práticas de sociabilidade e de atividades culturais (em conjunto, estas modalidades contribuem com 59,5 \% da variância do eixo 'específico'). Em oposição, no lado inferior do eixo, podemos encontrar experiências definidas por contribuições significativas de diferentes práticas de sociabilidade e de atividades culturais que são desenvolvidas de modo intenso ou moderado ( 25,7 \% de contribuição para a variância do eixo).

Tabela 4 : 24 modalidades significativas representadas na Figura 3 e respetivas contribuições para o eixo 2

\begin{tabular}{|l|l|l|}
\hline & \multicolumn{2}{|l|}{ Contribuições } \\
\hline Variáveis e modalidades & \multicolumn{2}{|l|}{ Eixo 2 } \\
\hline (R) Dores insuportáveis por causa do acidente de trabalho (AT) & Cima & Baixo \\
\hline 'Tenho dores insuportáveis' + & 2.9 & \\
\hline (R) Tenho medo por causa AT & & \\
\hline
\end{tabular}




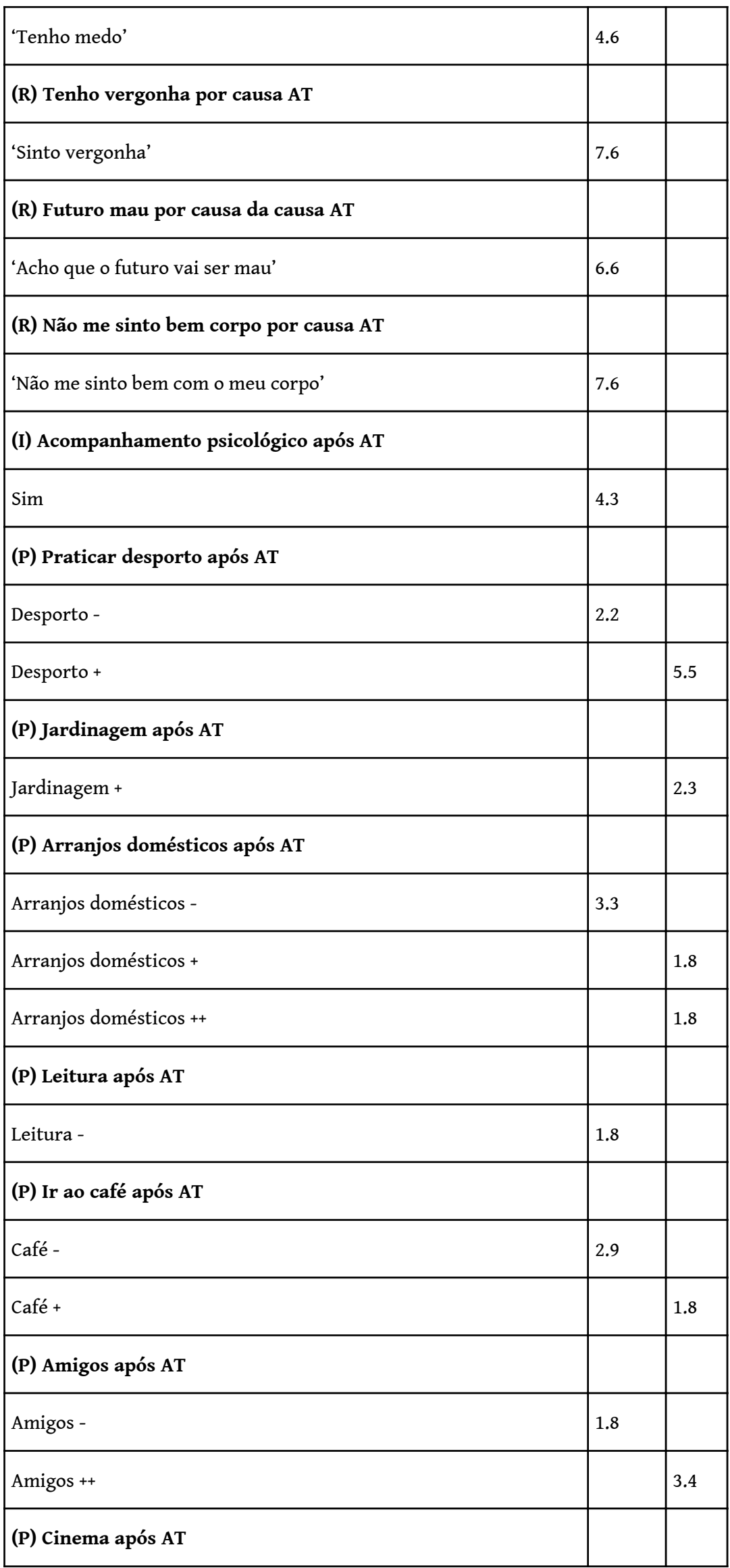




\begin{tabular}{|l|l|l|}
\hline Cinema - & 2.9 & \\
\hline Cinema + & & 4.3 \\
\hline (P) Andar a pé após AT & & \\
\hline Andar a pé - & 5.3 & \\
\hline Andar a pé + & & 2.9 \\
\hline Andar a pé ++ & & 1.9 \\
\hline (I) Apoio ACT após AT & & \\
\hline ACT + & 2.3 & \\
\hline (I) Apoio Segurança Social após AT & 3.4 & \\
\hline Seg. Social + & 59.5 & 25.7 \\
\hline Total das contribuições & & \\
\hline
\end{tabular}

Figura 3 : ACM 'específica'. Nuvem das categorias - 24 modalidades que contribuem mais para o eixo 2.

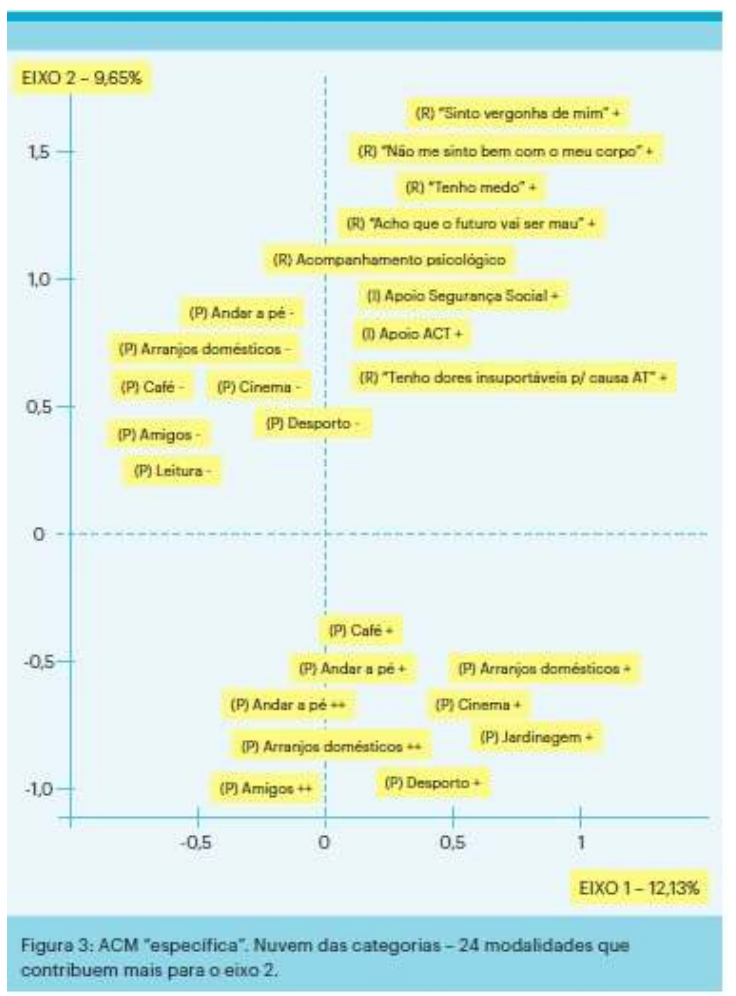

15 Em síntese, entre os trabalhadores industriais inquiridos podemos, assim, identificar modalidades alternativas de experiência do pós-sinistro. Um primeiro eixo estrutura estas experiências de acordo com a importância de uma articulação entre o desenvolvimento de uma sociabilidade moderada, um enquadramento institucional 
significativo e sentimentos de dor. Tais modalidades de experiência do pós-sinistro contrastam com as que se definem por um cenário alternativo marcado pela ausência de sociabilidade e de enquadramento institucional. Um segundo eixo configura as experiências do pós-sinistro em torno de um quadro relacional onde se aliam sentimentos de medo, vergonha e dor, acompanhados por seguimento psicológico, por apoios institucionais específicos e pela ausência de atividade sociabilitária. Estas modalidades distinguem-se das experiências marcadas pelo envolvimento moderado ou intenso em matéria de sociabilidade e de atividades culturais.

A informação sobre variáveis suplementares é apresentada nas Tabelas 2 a 4 e nas Figuras 4 a 6. Como se verifica nestas, as experiências do pós-acidente de trabalho dos trabalhadores industriais em estudo podem também ser descritas com a ajuda de informação suplementar.

17 A Tabela 5 e a Figura 4 apresentam informação sobre os grupos etários destes trabalhadores industriais. As experiências do pós-acidente de trabalho identificadas no eixo 1 são caraterizadas por diferenças etárias 'notáveis' : adultos-jovens (35-44 anos), no lado direito do eixo, opõem-se aos trabalhadores industriais mais velhos (>65) e aos adultos-envelhecidos (55-64), no lado esquerdo. 0 segundo eixo específico também é marcado por desvios etários 'notáveis' : neste caso, os adultos (45-54 anos) e adultosjovens (35-44) diferenciam-se dos mais novos (25-34) dos trabalhadores industriais que se posicionam na parte inferior da figura.

Tabela 5 : Frequências absolutas e coordenadas nos eixos 1 e 2 da informação sobre a idade dos respondentes

\begin{tabular}{|l|l|l|l|}
\hline & & \multicolumn{2}{|l|}{ Coordenadas } \\
\hline Grupos etários : modalidades & Frequências absolutas & Eixo 1 & Eixo 2 \\
\hline $25-34$ & 9 & 0,27 & $-0,60$ \\
\hline $35-44$ & 42 & 0,40 & 0,10 \\
\hline $45-54$ & 58 & 0,04 & 0,08 \\
\hline $55-64$ & 63 & $-0,26$ & $-0,02$ \\
\hline$>=65$ & 9 & $-0,47$ & $-0,07$ \\
\hline Sem informação & 3 & Não representada & Não representada \\
\hline
\end{tabular}


Figura 4 : ACM 'Específica'. Variáveis suplementares : Grupos etários dos trabalhadores industriais inquiridos nos eixos 1 e 2

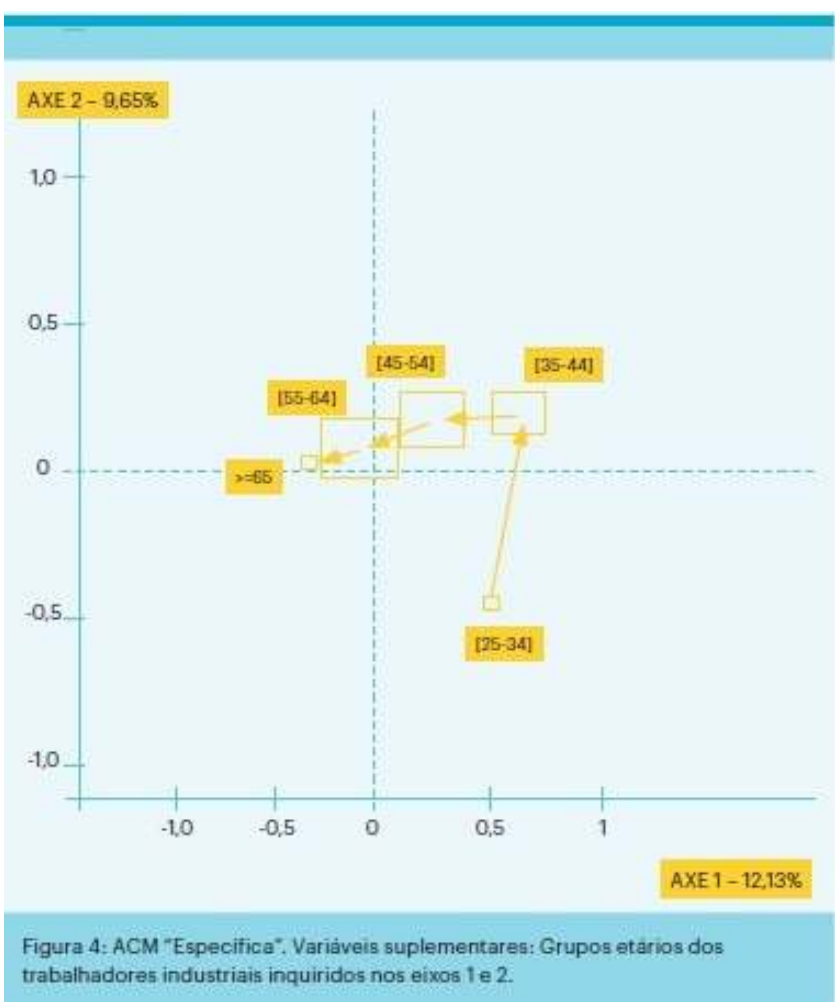

18 A Tabela 6 e a Figura 5 apresentam informação sobre a relação entre os resultados das variáveis ativas da ACM 'específica' e o capital cultural institucionalizado dos inquiridos, aqui medido em títulos escolares e em anos de educação formal (Bourdieu, 1979a : 21-28; 1979b : 5-6). As experiências dos trabalhadores industriais documentadas no primeiro eixo são marcadas por desvios 'notáveis' de capital cultural, opondo os trabalhadores com 12 anos de escolaridade aos que têm quatro anos ou menos. As diferenças de capital cultural no segundo eixo não são significativas.

Tabela 6 : Frequências absolutas e coordenadas nos eixos 1 e 2 da informação sobre o capital cultural dos trabalhadores industriais inquiridos

\begin{tabular}{|l|l|l|l|}
\hline & & \multicolumn{2}{|l|}{ Coordenadas } \\
\hline \multirow{2}{*}{$\begin{array}{l}\text { Capital cultural : modalidades } \\
4 \text { anos ou menos } \\
6 \text { anos } \\
9 \text { anos }\end{array}$} & Frequências absolutas & Eixo 1 & Eixo 2 \\
\hline $\begin{array}{l}12 \text { anos } \\
\text { Sem informação }\end{array}$ & 48 & $-0,32$ & 0,14 \\
\cline { 2 - 5 } & 43 & $-0,07$ & 0,07 \\
\cline { 2 - 5 } & 33 & 0,18 & $-0,11$ \\
\cline { 2 - 5 } & 2 & 0,36 & $-0,28$ \\
\hline
\end{tabular}


Figura 5 : ACM 'Específica'. Variáveis suplementares : Capital cultural dos trabalhadores industriais inquiridos nos Eixos 1-2

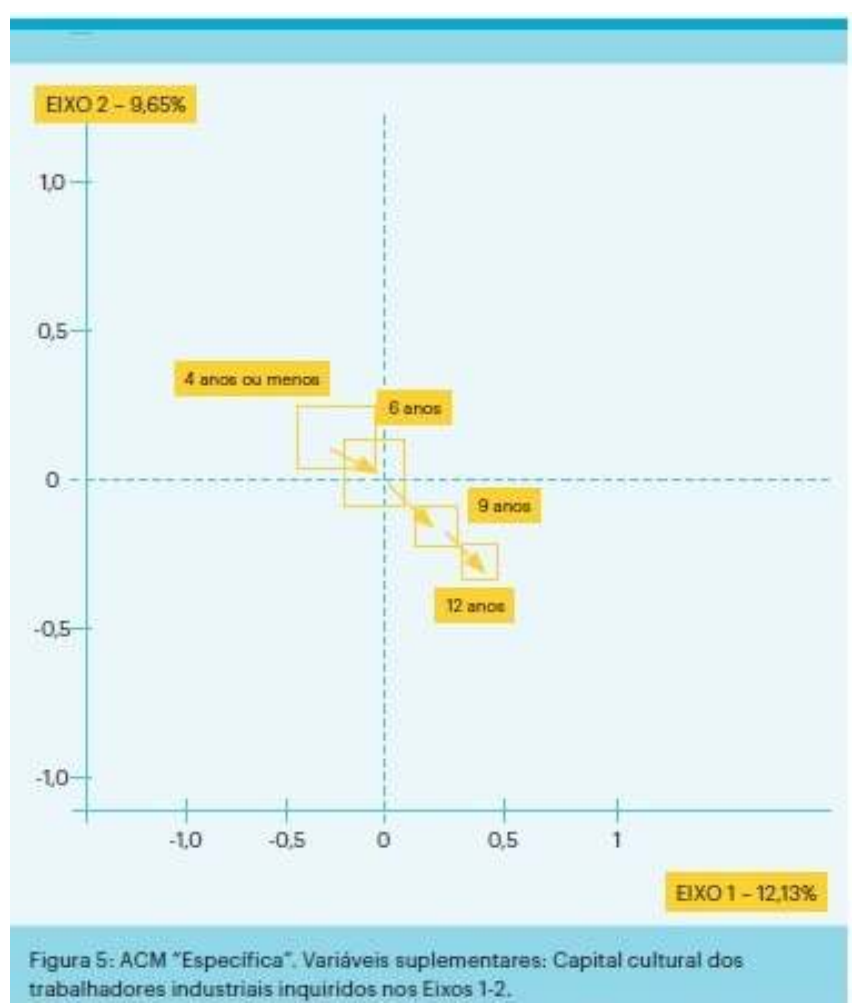

19 A Tabela 7 e a Figura 6 apresentam informação sobre os grupos de atividade económica dos trabalhadores industriais inquiridos. Neste caso, e uma vez que os trabalhadores partilhavam a mesma classe social, uma análise que consagrasse uma divisão interna da mesma dotada de maior detalhe apresentou-se como analiticamente pertinente, o que impede a presunção de uma homogeneidade estrita desta classe e, pelo contrário, salienta clivagens internas dotadas de significado sociológico. Assim, os grupos de atividade económica dos trabalhadores industriais foram usados para caraterizar as experiências em estudo. No eixo 1 não foi possível identificar diferenças significativas entre os grupos de atividade económica dos trabalhadores. Contudo, o eixo 2 envolve um desvio 'notável' entre trabalhadores com profissões no comércio e reparações, no setor dos transportes e da construção e aqueles que, na região inferior da figura, possuem profissões no setor da eletricidade e do gás.

Tabela 7 : Frequências absolutas e coordenadas nos eixos 1 e 2 da informação sobre os grupos de actividade económica dos trabalhadores industriais inquiridos

\begin{tabular}{|c|c|c|c|}
\hline & & \multicolumn{2}{|c|}{ Coordenadas } \\
\hline $\begin{array}{l}\text { Grupos de atividade económica: } \\
\text { modalidades }\end{array}$ & $\begin{array}{l}\text { Frequências } \\
\text { absolutas }\end{array}$ & Eixo 1 & Eixo 2 \\
\hline Indústria transformadora & 44 & 0,07 & 0,02 \\
\hline Indústria do gás e da eletricidade & 20 & 0,08 & $-0,50$ \\
\hline Comércio e reparações & 46 & $-0,20$ & 0,01 \\
\hline
\end{tabular}

Laubrasas, situacẽos ํo 1 | 2018 


\begin{tabular}{|l|l|l|l|}
\hline 12 & $-0,21$ & 0,33 \\
\hline 47 & 0,18 & 0,14 \\
\hline 15 & $\begin{array}{l}\text { Não } \\
\text { representada }\end{array}$ & $\begin{array}{l}\text { Não } \\
\text { representada }\end{array}$ \\
\hline
\end{tabular}

Figura 6 : ACM 'Específica'. Variáveis suplementares : Grupos de atividade económica dos trabalhadores industriais inquiridos nos Eixos 1-2

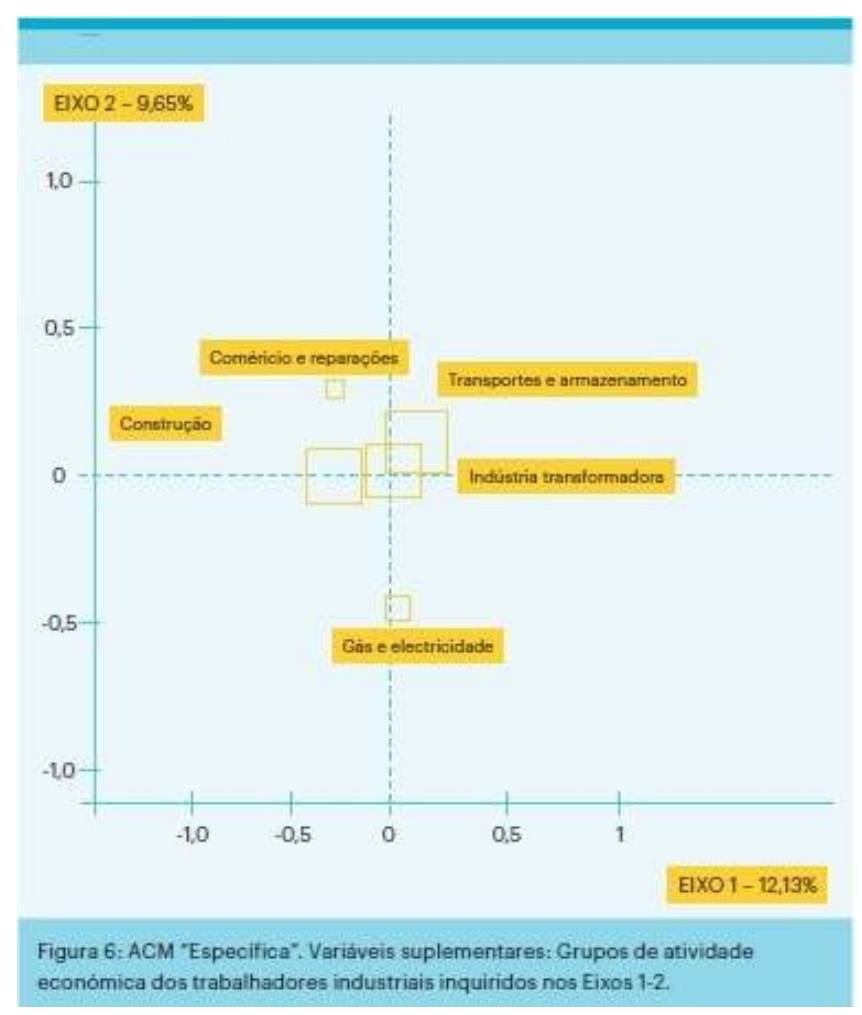

\section{Notas finais}

Os resultados de uma leitura relacional da informação aqui reunida merecem uma atenção sociológica. A incidência de acidentes de trabalho define-se por uma configuração plural de propriedades e práticas de natureza social. Tal como pudemos demonstrar com a ajuda de uma análise de correspondências múltiplas " específica ", as consequências pós-sinistro implicam, para os trabalhadores aqui inquiridos, experiências que têm características fenomenológicas e institucionais distintas, bem como relevantes inscrições sociais. Neste estudo, mostrámos como, num primeiro momento, o contraste entre sociabilidade moderada e cobertura institucional ativa e, por outro lado, desvinculação institucional e interacional configura a experiência vivida após-acidente dos trabalhadores industriais inquiridos. Num segundo momento, mostrámos que a prevalência de sofrimento vivido, de um lado, e a adesão a atividades culturais e de sociabilidade, de outro, caracteriza outro vetor destas experiências. 
21 Além de patentearem diferenças de idade com características específicas, as experiências dos trabalhadores aqui em questão parecem ser informadas por propriedades sociais com significado. Documentadas a respeito do primeiro eixo, as divisões que levam a proteção institucional e sociabilidade de média intensidade, por um lado, e a desvinculações, por outro, são compreensíveis a partir das diferenças em termos de diferenças culturais : a modalidade de capital cultural dominante entre os trabalhadores industriais (“ 12 anos ») constitui uma propriedade daqueles que têm proteção institucional e sociabilidade moderada, enquanto a desvinculação é característica daqueles que possuem as formas mais frágeis de capital cultural (“ Quatro anos ou menos »). Em particular, esta modalidade da experiência pós-sinistro constituída por desvinculação sociabilitária e ausência de cobertura institucional significativa - tem, portanto, uma relação com a escassez de informação e competências culturais (títulos e qualificações, por exemplos). Dada a importância que tem a informação, o acesso a ela e a sua utilização expedita, na superação das contrariedades que se sucedem aos acidentes de trabalho, esta relação entre escassez de cobertura institucional e frágil capital cultural atesta um impasse. Estas constatações merecem uma atenção da parte das políticas públicas comprometidas em solucionar tais desafios sociais e individuais.

Fomos ainda capazes de mostrar que as divisões que exprimem sofrimento físico e social e, por contraste, as que definem uma sociabilidade ativa e diversificada, estão associadas a distintos grupos de atividade económica : trabalhadores do comércio, dos transportes e da construção tendem a estar mais expostos ao sofrimento aqui representado ; práticas de sociabilidade mais expressivas e abrangentes são mais típicas de trabalhadores do gás e eletricidade. Para além da natureza do acidente e do impacto socialmente construído que tem sobre a vida destes trabalhadores sinistrados, a configuração deste segundo eixo específico lembra-nos da importância que têm as inscrições económicas destes trabalhadores (dimensão da empresa e a sua relação com as qualificações da mão-de-obra, por exemplo) e as regulações económica e política dos seus setores de atividade (presença do Estado, tradições sindicais, etc.). A composição económica e sócio-política que parece sustentar este segundo eixo específico, tal como os questionamentos adicionais sobre as implicações deste exercício para o estudo das classes laboriosas e as suas relações com as instituições, parecem justificar que se continue o estudo relacional aqui apresentado de maneira preliminar.

\section{BIBLIOGRAFIA}

Benzécri, J.-P. (1969). 'Statistical analysis as a tool to make patterns emerge from data' (pp. 35-74), In: S. Watanabe (Ed.), Methodologies of Pattern Recognition, New York: Academic Press.

Bourdieu, P. (1979a). La Distinction: critique sociale du jugement. Paris: Les Éditions de Minuit. Bourdieu, P. (1979b). Les Trois États du Capital Culturel, Actes de la Recherche en Sciences Sociales, 30, 3-6. 
Daubas-Letourneux, V. (2012). Les accidents du travail: une catégorie limpide? Une comparaison franco-suisse (pp. 125-138), in: Véronique Daubas-Letourneux et al, Santé au travail: approches critiques, Paris, La Découverte.

Dembe, A. (2001). The social consequences of occupational injuries and illnesses, American Journal of Industrial Medicine, 40(4), 403-417.

Desmarez, P., Godin, I. \& Renneson, B. (2007). L'impact des accidents du travail sur le statut socioéconomique des victimes, Le Travail Humain, 2, volume 70, 127-152.

Dimopoulou, I., Anthi, A., Mastora, Z. \& Roussos, C. (2004). Health-Related Quality of Life and Disability in Survivors of Multiple Trauma One Year After Intensive Care Unit Discharge, American Journal of Physical Medicine \& Rehabilitation, 83(3), 171-176.

Ferrão, J. (2017). Ruralidades e Território no Capitalismo Contemporâneo. Uma Visão de Longa Duração sobre Portugal (pp. 229-245), In : Fernando Luís Machado et al, Sociologia e Sociedade : Estudos de Homenagem a João Ferreira de Almeida, Lisboa, Mundos Sociais.

Franche, R.-L. \& Krause, N. (2002). Readiness for Return to Work Following Injury or Illness: Conceptualizing the Interpersonal Impact of Health Care, Workplace, and Insurance Factors, Journal of Occupational Rehabilitation, 12 (4), 233-256.

Ghisi, M., Novara, C., Buodo, G., Kimble, M., Scozzari, S., Di Natale, A., Sanavio, E., Palomba, D. (2013). Psychological Distress and Post-Traumatic Symptoms Following Occupational Accidents, Behavioural Sciences, 3, 587-600.

Holmes, S. (2013). Fresh Fruit, Broken Bodies: Migrant Farmworkers in the United States, Berkeley, University of California Press.

Keller, S. D. (2001). Quantifying social consequences of occupational injuries and illnesses: state of the art and research agenda, American Journal of Industrial Medicine, 40(4), 438-451.

Krieger, N. (2005). Embodiment: a conceptual glossary for epidemiology, Journal of Epidemiology and Community Health, 59 (5), 350-355.

Lax, M. B. \& Klein, R. (2008). More than meets the eye: social, economic, and emotional impacts of work-related injury and

illness, New solutions: a journal of environmental and occupational health policy, 18(3), 343-360.

Le Roux, B. \& Rouanet, H. (2010). Multiple Correspondence Analysis. London: Sage. https:// doi.org/10.4135/9781412993906 .

Lenoir, R. (1980). La notion d'accident de travail: un enjeu de lutes, Actes de la Recherche en Sciences Sociales, 32-33, 77-88.

Lock, M. \& Kaufert, P. (2001). Menopause, Local Biologies and Cultures of Aging. Margaret Lock and Patricia Kaufert. American Journal of Human Biology, 13(4): 494-504.

Lovelock, K. (2012). The injured and diseased farmer: occupational health, embodiment and technologies of harm and care, Sociology of Health \& Illness, 34(4), 576-590.

Mauss, M. (1969). Débat sur les rapports entre la sociologie et la psychologie, in: Marcel Mauss, Oeuvres. 3. Cohésion sociale et division de la sociologie. Paris : Les Éditions de Minuit, 298-302.

Monteiro, B. (2014). Frágil como o mundo. Etnografia do quotidiano operário, Porto, Edições Afrontamento.

Pinto, J. M. (2000). Flexibilidade, segurança e identidades sócio-profissionais. Cadernos de Ciências Sociais, 19/20, 5-37. 
Pinto, J. M. \& Queiroz, M. C. (1990). Lugares de Classe e Contextos de Aprendizagem Social, Cadernos de Ciências Sociais, 8/9, 131-143.

Pereira, V. B. (2011). Experiencing unemployment: The roles of social and cultural capital in mediating economic crisis, Poetics, 39 (6), 469-490.

Scheper-Hughes, N. \& Lock, M. (1987). The Mindful Body: A Prolegomenon to Future Work in Medical Anthropology. Medical Anthropology Quarterly, 1(1), 6-41 https://doi.org/10.1525/maq. 1987.1.1.02a00020

Teasdale, T. W. \& Engberg, A. W. (2005). Psychosocial consequences of stroke: A long-term population-based follow-up, Brain injury, volume 19, 1049-1058.

Wacquant, L. (1998). Pugs at work: bodily capital and bodily labour among professional boxers, Body and Society, 1, 65-93.

\section{NOTAS}

1. Uma primeira versão deste texto foi apresentada oralmente na sessão de trabalho Rising Inequalities, realizado na Universidade de Aalborg (campus de Copenhaga), a 14 e 15 de setembro de 2017, organizado com o apoio do NOS-HS - The joint committee for Nordic research councils within the humanities and social sciences. Os autores gostariam de agradecer a Vegard Jarness (Universidade de Bergen) pelos comentários que realizou nessa altura sobre a apresentação. Os resultados aqui apresentados foram desenvolvidos a partir da pesquisa realizada no âmbito do supramencionado projeto «Regresso ao trabalho após acidente: superar obstáculos», promovido pela Associação Nacional de Deficientes Sinistrados no Trabalho, com o apoio do Instituto Nacional de Reabilitação. Para a consolidação do argumento e da abordagem deste artigo foram cruciais os debates desenvolvidos pelo projeto de investigação 'Novos terrenos para a construção: mudanças no campo português da construção e seus impactos nas condições de trabalho no século XXI' (PTDC/IVC-SOC/5578/2014-016621), que é financiado por Fundos FEDER através do Programa Operacional Competitividade e Internacionalização - COMPETE 2020 e por Fundos Nacionais através da FCT - Fundação para a Ciência e a Tecnologia. Os autores gostariam de agradecer ainda aos dois revisores anónimos que comentaram a versão inicialmente submetida para publicação.

2. Em síntese, o Nottingham Health Profile (NHP) mede o impacto das condições de saúde e de doença nas rotinas da vida quotidiana (entre outras, hábitos de prática ou de sono, emoções e dor, mobilidade física), cobrindo analiticamente a intensidade das distorções trazidas para os domínios do trabalho e do emprego, do trabalho de casa e da dinâmica familiar, ou das interações sociais e do lazer. Ainda que seja habitualmente aplicado para explorar os impactos da doença, o NHP também permite uma aproximação às expressões 'subjetivas' e aos sintomas de acontecimentos traumáticos e às consequências pós-ferimento, tais como derrames vasculares (Teasdale e Engberg, 2010) ou traumas múltiplos (Dimopoulou et al, 2004). A versão portuguesa utilizada foi a adaptada por investigadores da Faculdade de Economia da Universidade de Coimbra, Centro de estudos e Investigação em Saúde, sob responsabilidade de Pedro Lopes Ferreira, editada em 1997.

3. A Análise de Correspondências Múltiplas é um método estatístico especialmente vocacionado para fazer 'emergir padrões dos dados', de acordo com as formulações de um dos seus principais criadores, J.-P. Benzécri (1969). O procedimento toma por referência uma matriz que relaciona um número potencialmente alargado de 'Questões' com variáveis categoriais, apresentadas em coluna, e de 'Indivíduos', que escolheram apenas uma modalidade por questão, apresentados em linha. 0 método procura uma redução de informação a partir da definição de um espaço ótimo de 
menor dimensionalidade. Com recurso à elaboração de uma tabela disjuntiva, o método permite a definição de uma nuvem de indivíduos e de uma nuvem de categorias com a mesma dimensionalidade e variância (Le Roux e Rouanet, 2010: 5-10).

4. A ACM 'específica' desenvolve-se com recurso aos mesmos procedimentos de uma ACM básica, mas envolve a restrição da análise a categorias de referência de uma ou mais variáveis, sendo outras transformadas em modalidades passivas, que não entram, por isso, nos procedimentos de cálculo (Le Roux e Rouanet, 2010: 62-64).

\section{RESUMOS}

Este artigo apresenta resultados preliminares de um estudo sociológico sobre a experiência de sofrimento físico e social de trabalhadores portugueses que sofreram um acidente de trabalho. Por via da combinação entre métodos distintos, usando um inquérito extensivo de uma amostra nacional de trabalhadores sinistrados e entrevistas em profundidade, este artigo identifica as principais caraterísticas que definem a experiência vivida dessas dores e explora, a partir da Análise de Correspondências Múltiplas, as virtualidades concedidas por uma leitura relacional da informação em termos sociológicos. Além de mostrar que a desigualdade está intensamente associada à (re)produção de acidentes laborais, este artigo argumenta que as inscrições sociais e os dispositivos institucionais têm um contributo importante na definição das experiências de sofrimento pós-sinistro.

Este artículo presenta los resultados preliminares de un estudio sociológico sobre la experiencia física y social del sufrimiento en trabajadores portugueses que han tenido un accidente de trabajo. A través de la combinación de distintos métodos, utilizando una encuesta extensiva en una muestra nacional de trabajadores víctimas de accidentes y entrevistas en profundidad, este artículo identifica las principales características que definen la experiencia vivida de esos dolores. Asimismo, explora, a partir del Análisis de Correspondencia Múltiple, las virtualidades concedidas por una lectura relacional de la información en términos sociológicos. Además de mostrar que la desigualdad se encuentra intensamente asociada a la (re)producción de accidentes laborales, este artículo explica cómo las inscripciones sociales y los dispositivos institucionales contribuyen a la definición de las experiencias de sufrimiento tras el siniestro.

Cet article présente des résultats préliminaires d'une étude sociologique sur l'expérience de la souffrance physique et sociale des travailleurs portugais qui ont eu un accident de travail. À partir d'une combinaison de différentes méthodes, et en utilisant des résultats d'une enquête extensive d'un échantillon national de travailleurs sinistrés et des entretiens en profondeur, cet article identifie les principales caractéristiques de l'expérience vécue de ces douleurs et explore, à partir de l'usage de l'Analyse de Correspondances Multiples, les virtualités d'une lecture relationnelle de l'information en termes sociologiques. Outre la démonstration de l'importance de l'association entre les inégalités sociales et la (re)production des accidents de travail, l'article argumente que les positions sociales occupées par les agents et les dispositifs institutionnels ont une contribution importante dans la définition des expériences de la souffrance après le sinistre.

This paper discusses some preliminary results of a mix-method sociological study about the experience of physical and social suffering lived by Portuguese workers involved in work accidents. Based on an extensive survey to a national sample of injured workers and in-depth 
interviews, the paper identifies the major characteristics that define how such physical and social pain was experienced and explores, with the help of Multiple Correspondence Analysis, the gains obtained from a socio-relational reading of this information. Besides showing that inequality is heavily inscribed in the (re)production of work accidents, the paper argues that social inscriptions and institutional devices play a major role in the definition of the postaccident pain experiences felt by the surveyed injured workers.

\section{ÍNDICE}

Mots-clés: post-accident, incorporation, douleur, divisions sociales, expérience vécue Palabras claves: pos-accidente, incorporación, dolor, divisiones sociales, experiencia vivida Palavras-chave: pós-acidente, incorporação, dor, divisões sociais, experiência vivida Keywords: post-accident, embodiment, pain, social divisions, lived experience

\section{AUTORES}

\section{BRUNO MONTEIRO}

Instituto de Sociologia da Universidade do Porto Faculdade de Letras da Universidade do Porto Via Panorâmica s/n 4150-564 - Porto bjrmonteiro@gmail.com

\section{VIRGÍLIO BORGES PEREIRA}

Departamento de Sociologia da Faculdade de Letras e Instituto de Sociologia da Universidade do Porto Faculdade de Letras da Universidade do Porto Via Panorâmica s/n 4150-564 - Porto jpereira@letras.up.pt 